\title{
ECONOMETRICA
}

Efficiency in the Optimum Supply of Public Goods

Author(s): Lawrence J. Lau, Eytan Sheshinski and Joseph E. Stiglitz

Source: Econometrica, Vol. 46, No. 2 (Mar., 1978), pp. 269-284

Published by: The Econometric Society

Stable URL: http://www.jstor.org/stable/1913900

Accessed: 01/05/2013 15:21

Your use of the JSTOR archive indicates your acceptance of the Terms \& Conditions of Use, available at http://www.jstor.org/page/info/about/policies/terms.jsp

JSTOR is a not-for-profit service that helps scholars, researchers, and students discover, use, and build upon a wide range of content in a trusted digital archive. We use information technology and tools to increase productivity and facilitate new forms of scholarship. For more information about JSTOR, please contact support@jstor.org. 


\title{
ECONOMETRICA
}

\section{EFFICIENCY IN THE OPTIMUM SUPPLY OF PUBLIC GOODS ${ }^{1}$}

\author{
By Lawrence J. Lau, Eytan Sheshinski, and Joseph E. Stiglitz
}

In this paper we are concerned with the following question: in any economy with several public goods, what are the conditions under which the conventional optimality rule of equality between the sum of marginal rates of substitution and the marginal rate of transformation still holds even in the presence of distortionary taxation? Two cases are considered. In the first case, the taxes may be arbitrary. In the second case, the taxes are optimally chosen.

\section{INTRODUCTION}

WHEN THE GOVERNMENT'S production of public goods is financed by distortionary taxes, the conventional optimality rule of equality between the sum of marginal rates of substitution and the marginal rate of transformation (Samuelson [13]) has to be modified so as to take account of the excess-burden created by the means of finance. The importance of this modification has already been recognized by Pigou [10], and has recently been treated formally by Atkinson and Stern [1]. These authors have examined the question of whether the optimum output levels of public goods financed by distortionary taxation are larger or smaller than their levels in the full optimum with lump-sum taxation. One expects intuitively that with distortionary taxation the conventional rule will overestimate the net benefits of public goods, but it has been shown that dependence of private consumption, and hence of tax revenue, on the supply of public goods, may reverse the intuitive conclusion.

In general, varying the supply of a public good will vary the demand for private goods (or the supply of factors), thereby varying government revenues. There is thus a fundamental interdependence between decisions about the relative quantities supplied of various public goods and the structure of taxation used to finance these goods. As a consequence, in general the marginal rate of substitution between two public goods is not equal at the optimum to their marginal rate of transformation (the ratio of producer prices).

In this paper we are concerned with the following question: in an economy with several public goods, under what circumstances does the conventional optimality rule of equality between marginal rates of transformation and substitution still hold, even in the presence of distortionary taxation? This question is important because if the conventional optimality rule holds, it is possible to

\footnotetext{
${ }^{1}$ This work was supported by National Science Foundation Grant SOC74-11446-A01 and SOC74-22182 at the Institute for Mathematical Studies in the Social Sciences, Stanford University. Sheshinski's work was partially supported by a grant by the Urban Institute to the Project on Efficiency of Decision Making in Economic Systems at Harvard University. We are grateful to Kenneth Arrow for pointing out an error in an earlier draft and to Avinash Dixit, Franklin Fisher, Frank Hahn, and Nicholas Stern for helpful comments.

The first version of this paper appeared as Technical Report No. 201 (April, 1976), Institute for Mathematical Studies in the Social Sciences, Stanford University.
} 
achieve a degree of decentralization in the optimum allocation of expenditures among various public goods. Specifically, given the total public goods budget, it is possible to determine the optimum allocation of expenditures among the various public goods independently of the magnitudes of the particular taxes (or subsidies) on the private goods that are used to finance them.

This independence is of importance for a number of reasons. In principle, decisions concerning taxation, distribution, and expenditure should be made simultaneously. In practice, however, different agencies within the government are concerned with the different governmental functions. Thus, Musgrave's [9] conventional division of the branches of the government may be thought of as more than just an analytical distinction. On the other hand, the sense in which the different branches can carry on their business separately from one another is not made clear in Musgrave (or in most of the subsequent literature), and the conditions under which various schemes of decentralization will lead to a full optimum are not known.

We shall show that under certain circumstances optimality is preserved by decentralization. In that case, the task of the "office of public goods allocation" is greatly simplified: it takes its budget, and "all" it has to do is to ascertain the individuals' marginal rates of substitution between any pair of public goods. Progress towards solving the problem of the revelation of preferences for public goods has recently been made (see, e.g., Groves and Ledyard [7] and Green and Laffont [6]). In this paper we shall assume that individual preferences are known to the government.

In some cases which admit decentralization in the above sense, the marginal rates of substitution will vary as the taxes vary, in others they will not. This distinction should affect the iterative procedures adopted to reach an optimum, but not the optimum conditions themselves.

The basic question of this paper is examined against two alternative assumptions. First we inquire about the conditions under which the allocative independence of production of public goods and taxation holds for any arbitrary set of taxes. It is shown that for this to hold, it is necessary and sufficient that the partial derivative of the demand of each private taxable.good with respect to the vector of quantities of public goods be proportional to the marginal utility of the vector of quantities of public goods. Second, we analyze how the previous answer changes when tax levels are chosen optimally (so as to minimize the dead-weight loss). We are able to characterize the set of indirect utility functions for which this is the case. As expected, the assumption of optimum taxation widens considerably the possibilities for independent optimum expenditure and tax decisions. Thus with regard to the class of utility functions which admits efficiency, we provide a number of sufficient conditions, none of which requires the proportionality conditions as in the arbitrary excise tax case. Obviously, the stronger condition suffices also when taxes are at their optimum level. As a special case of practical importance, we prove that among all direct additive utility functions, the only utility functions which "work" are those with constant and identical elasticities of substitution among the taxable commodities. 
Although the analysis of this paper is couched in terms of a representative individual, as usual, the validity of the results extends to the case of many individuals provided there are optimum lump-sum redistributions. ${ }^{2}$ When such redistributions are not available, our results provide simple conditions under which the allocative branch still maintains Pareto optimality, subject to a taxation constraint in the supply of public goods, thus providing a separation not only between the taxation and expenditure branches of the government, but also between the expenditure and redistribution branches.

The plan of the paper is as follows. Section 2 sets up the consumer maximization conditions. Sections 3 and 4 treat the cases of arbitrary and optimum taxes, respectively. Section 5 presents a proof that the constant-elasticity-of-substitution utility function is the only additive utility function which satisfies the conditions for efficiency when taxes are chosen optimally but not when taxes are arbitrary.

\section{CONSUMER UTILITY MAXIMIZATION}

We distinguish between three groups of goods: nontaxable private goods, taxable private goods, and public goods. Let $L=\left(L_{1}, L_{2}, \ldots, L_{s}\right), X=$ $\left(X_{1}, X_{2}, \ldots, X_{n}\right)$, and $Z=\left(Z_{1}, Z_{2}, \ldots, Z_{m}\right)$ be the vectors of total quantities of these goods, respectively. The dimensions $s$ and $n$ are arbitrary but not less than one and the dimension $m$ is arbitrary but not less than two. ${ }^{3}$ There is one consumer whose preferences are represented by the utility function $U=$ $U(L, X, Z){ }^{4}$

The consumer is assumed to maximize utility with respect to the quantities of the private goods, taxable and nontaxable, subject to a given vector of public goods, given consumer prices (which include the excise taxes if any), and total income. Thus, his problem is

$$
\begin{aligned}
& \max _{L, X} U(L, X, Z) \\
& \text { subject to } w^{* \prime} L+q^{* \prime} X=I
\end{aligned}
$$

where $w^{*}$ is the vector of nominal consumer prices of the nontaxable private goods, $q^{*}$ is the vector of nominal consumer prices of the taxable private goods, and $I$ is nominal total income, which may depend on $w^{*}$. For example, $I$ may be equal to $w_{1}^{*} \bar{L}$, where $w_{1}^{*}$ is the wage rate and $\bar{L}$ is the endowment of leisure. We define normalized price vectors $w=w^{*} / I$ and $q \equiv q^{*} / I$. Then the budget

\footnotetext{
${ }^{2}$ This has been shown in the classic paper by Boiteux [3]. Evidently, the possibility of lump-sum redistributions may appear to be incompatible with the assumption that the production of public goods is financed by distortive taxes.

${ }^{3}$ When $s=0$, the solution to (4.1)-(4.3) below can be shown to have the form $q=\mu p$, where $\mu$ a scalar, and the supply of public goods satisfies the efficiency condition (3.8). Obviously, this is the case of lump-sum taxation, which entails efficiency in the supply of public goods.

${ }^{4}$ In the case of $N$ identical individuals, utility depends on per-capita consumption $U[(L / N),(X / N), Z]$. Since $N$ is fixed throughout, we set $N=1$.
} 
constraint becomes

$$
w^{\prime} L+q^{\prime} X=1 \text {. }
$$

Under suitable regularity conditions, there exists an indirect utility function $V(w, q, Z)$ which is the maximized value of utility for given values of $w, q$, and $Z$. In particular, the optimum quantities of $L$ and $X$ are given by Roy's Identity [12]:

$$
L=\frac{\frac{\partial V}{\partial w}}{\left(w^{\prime} \frac{\partial V}{\partial w}+q^{\prime} \frac{\partial V}{\partial q}\right)},
$$

$$
X=\frac{\frac{\partial V}{\partial q}}{\left(w^{\prime} \frac{\partial V}{\partial w}+q^{\prime} \frac{\partial V}{\partial q}\right)} .
$$

\section{THE CASE WITH ARBITRARY EXCISE TAXES}

The government is assumed to maximize the consumer's utility with respect to the quantities of public goods $Z$, taking the private utility-maximizing behavior of the consumer, the producer prices of the taxable goods, $p$, the excise taxes, $(q-p)$, the prices of nontaxable goods, $w$, and the producer prices of the public goods, $r$, as given, and subject to a balanced budget constraint. ${ }^{5}$ That is:

$$
\max _{Z} V(w, q, Z)
$$

subject to

$$
(q-p)^{\prime} X-r^{\prime} Z=0 .
$$

As before, both $p$ and $r$ are prices normalized by total income. Correspondingly, $q-p$ is the vector of normalized excise taxes.

To solve the maximization problem, we may form the Lagrangian,

$$
L(w, q, Z, \lambda)=V(w, q, Z)-\lambda\left[(q-p)^{\prime} X-r^{\prime} Z\right]
$$

where $\lambda$ is a scalar. The first-order necessary conditions for a maximum with respect to $Z$ and $\lambda$ are:

$$
\begin{aligned}
& \frac{\partial L}{\partial Z}=\frac{\partial V}{\partial Z}-\lambda\left[\frac{\partial}{\partial Z}(q-p)^{\prime} X-r\right]=0 ; \\
& \frac{\partial L}{\partial \lambda}=(q-p)^{\prime} X-r^{\prime} Z=0 .
\end{aligned}
$$

${ }^{5}$ Note that by treating producer prices as given, we assume implicitly that the unit production costs are independent of the level of production which implies zero profits in the private sector. The latter is a basic condition in the decentralization results of Diamond and Mirrlees [4]. 
Condition (3.4), due to Boiteux[3] and Diamond and Mirrlees [4], has a natural interpretation. It states that, for each public good, marginal benefits should be proportional to marginal production costs less the additional tax revenue generated by an increase in the quantity of that good.

We now state our basic question: what are the conditions on $V(w, q, Z)$ such that whenever equations (3.4) and (3.5) hold, $\partial V / \partial Z$ is proportional to $r$ for arbitrary $w, q, p$, and $r$ ? Equivalently, in terms of the marginal rates of substitution, when do equations (3.4) and (3.5) imply that:

$$
\frac{\frac{\partial V}{\partial Z_{i}}}{\frac{\partial V}{\partial Z_{1}}}\left(=\frac{\frac{\partial U}{\partial Z_{i}}}{\frac{\partial U}{\partial Z_{1}}}\right)=\frac{r_{i}}{r_{1}}
$$

$$
(i=2,3, \ldots, m)
$$

for all public goods?

If $\partial V / \partial Z$ were to be proportional to $r$ at the optimum, then we have:

$$
\mu \frac{\partial V}{\partial Z}=r
$$

where $\mu$ is a scalar function of $w, q, p$, and $r$. Substituting equation (3.7) into equation (3.4), we obtain:

$$
\frac{\partial V}{\partial Z}-\lambda\left[\frac{\partial}{\partial Z}(q-p)^{\prime} X-\mu \frac{\partial V}{\partial Z}\right]=0
$$

or

$$
\frac{\partial V}{\partial Z}=\frac{\lambda}{1+\lambda \mu}\left[\frac{\partial}{\partial Z}(q-p)^{\prime} X\right]
$$

If, in addition, we assume that given $w, q$, and $p$, every value of $Z$ is potentially a solution of equations (3.4) and (3.5) which satisfies equation (3.6) for some choice of $r$, then equation (3.9) must hold for every value of $Z$. Thus, our objective is to characterize the class of indirect utility functions $V(w, q, Z)$ for which equation (3.9) holds identically for some $\mu$. In order for this to happen, it is necessary and sufficient that

$$
\begin{aligned}
\frac{\partial V}{\partial Z} & \sim \frac{\partial}{\partial Z}(q-p)^{\prime} X \\
& \sim \frac{\partial X}{\partial Z}(q-p)
\end{aligned}
$$

where $\sim$ denotes proportionality. But since $q$ and $p$ are both arbitrary, this implies that one must have, separately for each $i$,

$$
\frac{\partial V}{\partial Z} \sim \frac{\partial X_{i}}{\partial Z}
$$

$$
(i=1,2, \ldots, n)
$$


or, equivalently,

$$
\frac{\partial V}{\partial Z} \sim \frac{\partial q_{i} X_{i}}{\partial Z}
$$$$
(i=1,2, \ldots, n) \text {. }
$$

This condition may be interpreted as follows: the vector of marginal changes in the demand (or equivalently the budget share) of the $i$ th taxable commodity with respect to $Z$ must be proportional to the vector of marginal utilities of $Z$. Interestingly, by a Lemma in Goldman and Uzawa [5], this condition is equivalent to the condition that the demand functions can be written in the form:

$$
X_{i}(w, q, Z)=f_{i}(w, q, V(w, q, Z))
$$$$
(i=1,2, \ldots, n) \text {. }
$$

In terms of the indirect utility function, the condition implied by equation (3.12) is equivalent, through Roy's Identity, to

$$
\begin{array}{r}
\frac{\partial V}{\partial Z} \sim \frac{\partial}{\partial Z}\left(\frac{\frac{\partial V}{\partial q_{i}}}{w^{\prime} \frac{\partial V}{\partial w}+q^{\prime} \frac{\partial V}{\partial q}}\right) \\
\sim \frac{\partial}{\partial Z}\left(\frac{q_{i} \frac{\partial V}{\partial q_{i}}}{w^{\prime} \frac{\partial V}{\partial w}+q^{\prime} \frac{\partial V}{\partial q}}\right)
\end{array}
$$

$$
(i=1,2, \ldots, n)
$$$$
(i=1,2, \ldots, n) .
$$

The corresponding condition on the direct utility function may be derived as follows. We know that at the optimum $\partial V / \partial Z=\partial U / \partial Z$ by the envelope theorem. Also, at the optimum,

$$
\frac{q_{i} \frac{\partial V}{\partial q_{i}}}{w^{\prime} \frac{\partial V}{\partial w}+q^{\prime} \frac{\partial V}{\partial q}}=\frac{X_{i} \frac{\partial U}{\partial X_{i}}}{L^{\prime} \frac{\partial U}{\partial L}+X^{\prime} \frac{\partial U}{\partial X}}=q_{i} X_{i}
$$

Thus equations (3.15) and (3.16) imply that

$$
\begin{array}{r}
\frac{\partial U}{\partial Z} \sim \frac{\partial}{\partial Z}\left(\frac{X_{i} \frac{\partial U}{\partial X_{i}}}{L^{\prime} \frac{\partial U}{\partial L}+X^{\prime} \frac{\partial U}{\partial X}}\right) \\
\sim \frac{\partial}{\partial Z}\left(\frac{\frac{\partial U}{\partial X_{i}}}{L^{\prime} \frac{\partial U}{\partial L}+X^{\prime} \frac{\partial U}{\partial X}}\right)
\end{array}
$$$$
(i=1,2, \ldots, n)
$$ 
We shall now prove the following:

THEOREM 1: Condition (3.6) holds at the optimum (3.4)-(3.5) for any arbitrary vector $(q-p)$ if and only if the indirect utility function satisfies equation (3.15) or, equivalently, if and only if the direct utility function satisfies equation (3.17).

REMARK: A well-known example of a utility function which satisfies equation (3.17) is $U(L, X, Z)=U(L, X, f(Z))$ where $f(Z)$ is a real-valued function. It is immediately apparent that $X_{i}\left(\partial U / \partial X_{i}\right) /\left(X^{\prime}(\partial U / \partial X)+L^{\prime}(\partial U / \partial L)\right)$ depends on $Z$ only through $f(Z), i=1,2, \ldots, n$, and thus its derivative with respect to $Z$ must be proportional to $\partial U / \partial Z=(\partial U / \partial f)(\partial f / \partial Z)$. The indirect utility function which corresponds to this direct utility function is $V(w, q, f(Z))$ which, by the same argument, can be shown to satisfy equation (3.15).

Proof: Necessity has already been shown in the text. We need only show sufficiency. But if equation (3.15) holds for all $Z, w$, and $q$, it implies equation (3.12), which in turn implies equation (3.10). Substituting equation (3.10) into equation (3.4), we find that $\partial V / \partial Z \sim$ r. Q.E.D.

An indirect utility function which satisfies equation (3.15) but is not completely separable in $Z$ is

$$
V=V(w, q, H(Z, w))
$$

where $H(\cdot)$ is homogeneous of any degree (including zero) in $w$. Similarly, the direct utility function

$$
U=U(L, X, H(Z, L))
$$

where $H(\cdot)$ is homogeneous of any degree (including zero) in $L$, satisfies equation (3.17).

Finally, we give two examples of indirect utility functions which satisfy equation (3.15) but are not separable in $Z$. First,

$$
V=V\left(\frac{w}{q \alpha}, Z\right)
$$

where $q$ is a scalar variable $(n=1)$ and $w$ and $Z$ are vectors. It is easy to verify that

$$
\frac{\partial}{\partial Z}\left(\frac{\frac{\partial V}{\partial q}}{w^{\prime} \frac{\partial V}{\partial w}+q^{\prime} \frac{\partial V}{\partial q}}\right)=\frac{\partial}{\partial Z}\left(\frac{-\alpha}{1-\alpha} q^{-1}\right)=0 .
$$

Second,

$$
V=H(F(w, q), w, Z),
$$


where $H$ and $F$ are real-valued functions and $H$ is homogeneous of degree zero in $w$ for given $F$ and $Z$. Then

$$
\frac{\partial}{\partial Z}\left(\frac{\frac{\partial V}{\partial q_{i}}}{w^{\prime} \frac{\partial V}{\partial w}+q^{\prime} \frac{\partial V}{\partial q}}\right)=\frac{\partial}{\partial Z}\left(\frac{\frac{\partial F}{\partial q_{i}}}{w^{\prime} \frac{\partial F}{\partial w}+q^{\prime} \frac{\partial F}{\partial q}}\right)=0 .
$$

Proportionality is thus trivially satisfied in both cases.

Examples of direct utility functions may be similarly constructed.

\section{THE CASE WITH OPTIMUM EXCISE TAXES}

Suppose now that taxes are not predetermined, but chosen optimally; that is, the government maximizes utility with respect to both $q$ and $Z$. The first-order necessary conditions for a maximum are:

$$
\begin{aligned}
& \frac{\partial L}{\partial q}=\frac{\partial V}{\partial q}-\lambda\left[\frac{\partial}{\partial q}(q-p)^{\prime} X\right]=0 ; \\
& \frac{\partial L}{\partial Z}=\frac{\partial V}{\partial Z}-\lambda\left[\frac{\partial}{\partial Z}(q-p)^{\prime} X-r\right]=0 ; \\
& \frac{\partial L}{\partial \lambda}=(q-p)^{\prime} X-r^{\prime} Z=0 .
\end{aligned}
$$

Equation (4.1) may be rewritten as:

$$
\frac{\partial V}{\partial q}-\lambda\left[X+\frac{\partial X}{\partial q}(q-p)\right]=0
$$

or, by Roy's Identity (2.5),

$$
\frac{\partial X}{\partial q}(q-p)-\theta X=0
$$

where $\theta$ is a scalar. This is the familiar "Ramsey formula" for optimal taxation (Ramsey [11]).

As before, we seek conditions on $V(w, q, Z)$ such that, at the optimum,

$$
\mu \frac{\partial V}{\partial Z}=r
$$

which implies that equation (4.2) may be rewritten as:

$$
\frac{\partial V}{\partial Z}=\frac{\lambda}{1+\lambda \mu}\left[\frac{\partial X}{\partial Z}(q-p)\right] .
$$

Obviously, if $\partial X_{i} / \partial Z \sim \partial V / \partial Z, i=1,2, \ldots, n$, equation (4.7) is satisfied. However, we do not require equation (4.7) to hold for all possible $(q-p)$. 
Instead, we require it to hold only for the $(q-p)$ 's which are optimum, given $w, p$, and $r$. If, in addition, we assume that given $w$ and $Z$, every value of $q$ is potentially a solution of equations (4.3) and (4.4) for some choice of $p$, then one can solve from equation (4.4) for $p$ or equivalently for $(q-p)$ as that value for which a given $q$ is optimum

$$
(q-p)=\frac{1}{\lambda}\left[\frac{\partial X}{\partial q}\right]^{-1}\left(\frac{\partial V}{\partial q}-\lambda X\right)
$$

Substituting equation (4.8) into equation (4.7), we obtain:

$$
\frac{\partial V}{\partial Z}=\frac{1}{1+\lambda \mu}\left[\frac{\partial X}{\partial Z}\left[\frac{\partial X}{\partial q}\right]^{-1}\left(\frac{\partial V}{\partial q}-\lambda X\right)\right] .
$$

By Roy's Identity,

$$
X=\frac{\frac{\partial V}{\partial q}}{q^{\prime} \frac{\partial V}{\partial q}+w^{\prime} \frac{\partial V}{\partial w}} .
$$

Thus, equation (4.9) becomes:

(4.10) $\frac{\partial V}{\partial Z} \sim \frac{\partial X}{\partial Z}\left[\frac{\partial X}{\partial q}\right]^{-1} X$.

By direct computation,

$$
\begin{aligned}
\frac{\partial X}{\partial Z} & =\frac{\partial}{\partial Z}\left(\frac{\frac{\partial V}{\partial q}}{q^{\prime} \frac{\partial V}{\partial q}+w^{\prime} \frac{\partial V}{\partial w}}\right) \\
& =\frac{1}{q^{\prime} \frac{\partial V}{\partial q}+w^{\prime} \frac{\partial V}{\partial w}}\left[\frac{\partial^{2} V}{\partial Z \partial q}-\frac{1}{q^{\prime} \frac{\partial V}{\partial q}+w^{\prime} \frac{\partial V}{\partial w}}\left[\frac{\partial^{2} V}{\partial Z \partial q} q+\frac{\partial^{2} V}{\partial Z \partial w} w\right] \frac{\partial V^{\prime}}{\partial q}\right],
\end{aligned}
$$

$$
\frac{\partial X}{\partial q}=\frac{1}{q^{\prime} \frac{\partial V}{\partial q}+w^{\prime} \frac{\partial V}{\partial w}}\left[\frac{\partial^{2} V}{\partial q^{2}}-\frac{1}{q^{\prime} \frac{\partial V}{\partial q}+w^{\prime} \frac{\partial V}{\partial w}}\left[\frac{\partial V}{\partial q}+\frac{\partial^{2} V}{\partial q^{2}} q+\frac{\partial^{2} V}{\partial q \partial w} w\right] \frac{\partial V^{\prime}}{\partial q}\right]
$$

Thus,

$$
\begin{aligned}
{\left[\frac{\partial X}{\partial q}\right]^{-1}=\left(q^{\prime} \frac{\partial V}{\partial q}+w^{\prime} \frac{\partial V}{\partial w}\right) } & {\left[\frac{\partial^{2} V}{\partial q^{2}}\right.} \\
& \left.-\frac{1}{q^{\prime} \frac{\partial V}{\partial q}+w^{\prime} \frac{\partial V}{\partial w}}\left[\frac{\partial V}{\partial q}+\frac{\partial^{2} V}{\partial q^{2}} q+\frac{\partial^{2} V}{\partial q \partial w} w\right] \frac{\partial V^{\prime}}{\partial q}\right]^{-1} .
\end{aligned}
$$


The condition for efficiency then becomes:

$$
\begin{aligned}
\frac{\partial V}{\partial Z} \sim & \frac{\partial^{2} V}{\partial Z \partial q}\left[\frac{\partial^{2} V}{\partial q^{2}}-\frac{1}{q^{\prime} \frac{\partial V}{\partial q}+w^{\prime} \frac{\partial V}{\partial w}}\left[\frac{\partial V}{\partial q}+\frac{\partial^{2} V}{\partial q^{2}} q+\frac{\partial^{2} V}{\partial q \partial w} w\right] \frac{\partial V^{\prime}}{\partial q}\right]^{-1} \frac{\partial V}{\partial q} \\
& -\frac{1}{q^{\prime} \frac{\partial V}{\partial q}+w^{\prime} \frac{\partial V}{\partial w}}\left[\frac{\partial^{2} V}{\partial Z \partial q} q+\frac{\partial^{2} V}{\partial Z \partial w} w\right] \frac{\partial V^{\prime}}{\partial q} \\
\sim & {\left[\frac{\partial^{2} V}{\partial q^{2}}-\frac{1}{q^{\prime} \frac{\partial V}{\partial q}+w^{\prime} \frac{\partial V}{\partial w}}\left[\frac{\partial V}{\partial q}+\frac{\partial^{2} V}{\partial q^{2}} q+\frac{\partial^{2} V}{\partial q \partial w} w\right] \frac{\partial V^{\prime}}{\partial q}\right]^{-1} \frac{\partial V}{\partial q} } \\
& {\left[\frac{\partial^{2} V}{\partial q^{2}}-\frac{1}{q^{\prime} \frac{\partial V}{\partial q}+w^{\prime} \frac{\partial V}{\partial w}}\left[\frac{\partial V}{\partial q}+\frac{\partial^{\prime} \frac{\partial V}{\partial w}}{\partial q^{2}} q+\frac{\partial^{2} V}{\partial Z \partial \partial w} w+\frac{\partial^{2} V}{\partial Z \partial w} w\right] \frac{\partial V^{\prime}}{\partial q}\right] }
\end{aligned}
$$

Equation (4.14) constitutes the necessary and sufficient conditions on the utility function for efficiency in the optimum supply of public goods under the specified institutional arrangements. This is a set of non-linear partial differential functional equations in $V$ which we have not been able to solve explicitly. However, equation (4.14) is useful in proving sufficient conditions. That is, it can be used to verify whether a given class of utility functions admits of efficient supply of public goods when taxes are assumed to be chosen optimally. In addition, it can be used as the basis for testing empirically whether the observed consumption behavior is consistent with this type of utility function.

One set of sufficient conditions consists of the following two equations:

$$
\frac{\partial V}{\partial Z} \sim \frac{\partial^{2} V}{\partial Z \partial q}\left[\frac{\partial^{2} V}{\partial q^{2}}-\frac{1}{q^{\prime} \frac{\partial V}{\partial q}+w^{\prime} \frac{\partial V}{\partial w}}\left[\frac{\partial V}{\partial q}+\frac{\partial^{2} V}{\partial q^{2}} q+\frac{\partial^{2} V}{\partial q \partial w} w\right] \frac{\partial V^{\prime}}{\partial q}\right]^{-1} \frac{\partial V}{\partial q}
$$

and

$$
\frac{\partial V}{\partial Z} \sim\left[\frac{\partial^{2} V}{\partial Z \partial q} q+\frac{\partial^{2} V}{\partial Z \partial w} w\right]
$$

Obviously, equations (4.15) and (4.16) together imply equation (4.14). Again, we have not been able to solve equations (4.15) and (4.16) explicitly. However, using these equations, we can generate indirect utility functions which fail to satisfy the conditions in Theorem 1 but nevertheless satisfy equation (4.14) when the excise taxes are chosen optimally. In Theorem 2 we give a set of sufficient conditions for efficiency on the indirect utility function for this case. 
THEOREM 2: Condition (3.6) holds at the optimum (4.1)-(4.3) with optimum taxation if the indirect utility function has the form $V=V(w, H(w, q, Z))$, where $H$ is homogeneous of degree one in $q$ and in addition satisfies the conditions:

(i) $\left(\partial H / \partial q_{i}\right) /\left(\partial H / \partial q_{1}\right), i=2,3, \ldots, n$, is homogeneous of degree zero in $w$; and

(ii) $\left(\partial H / \partial Z_{i}\right) /\left(\partial H / \partial Z_{1}\right), i=2,3, \ldots, m$, is homogeneous of degree zero in $w$.

Before proving Theorem 2, we present the following Lemma 1 which we shall need in the proof of the Theorem:

LeMMA 1: Let $A$ be a real, symmetric nonsingular $n \times n$ matrix and a be a real $n \times 1$ vector; then

$$
\left[A-a a^{\prime}\right]^{-1}=A^{-1}-\frac{1}{\left(a^{\prime} A^{-1} a-1\right)} A^{-1} a a^{\prime} A^{-1}
$$

provided $a^{\prime} A^{-1} a-1 \neq 0$.

ProOF:

$$
\begin{aligned}
{\left[A-a a^{\prime}\right] } & {\left[A^{-1}-\frac{1}{\left(a^{\prime} A^{-1} a-1\right)} A^{-1} a a^{\prime} A^{-1}\right] } \\
& =I-\frac{1}{\left(a^{\prime} A^{-1} a-1\right)} a a^{\prime} A^{-1}-a a^{\prime} A^{-1}+\frac{a a^{\prime} A^{-1} a a^{\prime} A^{-1}}{\left(a^{\prime} A^{-1} a-1\right)} \\
& =I .
\end{aligned}
$$

Q.E.D.

Proof of Theorem 2: To see that this utility function satisfies equations (4.15) and (4.16), we first compute:

$$
\frac{\partial V}{\partial q}=\frac{\partial V}{\partial H} \cdot \frac{\partial H}{\partial q}
$$

$$
\frac{\partial^{2} V}{\partial q^{2}} q=\frac{\partial H}{\partial q} \frac{\partial^{2} V}{\partial H^{2}} \frac{\partial H^{\prime}}{\partial q} q+\frac{\partial V}{\partial H} \frac{\partial^{2} H}{\partial q^{2}} q=\left[\frac{\frac{\partial^{2} V}{\partial H^{2}} H}{\frac{\partial V}{\partial H}}\right] \frac{\partial V}{\partial q},
$$

$$
\frac{\partial^{2} V}{\partial q \partial w} w=\frac{\partial H}{\partial q} \frac{\partial^{2} V}{\partial H \partial w} w+\frac{\partial H}{\partial q} \frac{\partial^{2} V}{\partial H^{2}} \frac{\partial H^{\prime}}{\partial w} w+\frac{\partial V}{\partial H} \frac{\partial^{2} H}{\partial q \partial w} w .
$$

Homogeneity of degree zero of $\left(\partial H / \partial q_{i}\right) /\left(\partial H / \partial q_{1}\right)$ in $w$ implies that

$$
\frac{\partial^{2} H}{\partial q_{i} \partial w} \frac{w}{H_{i}}-\frac{\partial^{2} H}{\partial q_{1} \partial w} \frac{w}{H_{1}}=0
$$

$$
(i=2,3, \ldots, n)
$$

or equivalently,

$$
\frac{\partial^{2} H}{\partial q_{i} \partial w} w=\psi \frac{\partial H}{\partial q_{i}}
$$

$$
(i=1,2, \ldots, n)
$$


where $\psi$ is a scalar. Taking equations (4.19), (4.20), and (4.22) together, equation (4.15) becomes:

$$
\frac{\partial V}{\partial Z} \sim \frac{\partial^{2} V}{\partial Z \partial q}\left[\frac{\partial^{2} V}{\partial q^{2}}-\eta \frac{\partial V}{\partial q} \frac{\partial V^{\prime}}{\partial q}\right]^{-1} \frac{\partial V}{\partial q}
$$

where $\eta$ is a scalar. By Lemma 1, equation (4.23) may be rewritten as:

$$
\frac{\partial V}{\partial Z} \sim \frac{\partial^{2} V}{\partial Z \partial q}\left[\left[\frac{\partial^{2} V}{\partial q^{2}}\right]^{-1}-\eta^{*}\left[\frac{\partial^{2} V}{\partial q^{2}}\right]^{-1} \frac{\partial V}{\partial q} \frac{\partial V^{\prime}}{\partial q}\left[\frac{\partial^{2} V}{\partial q^{2}}\right]^{-1}\right] \frac{\partial V}{\partial q}
$$

where $\eta^{*}$ is another scalar. Since $\partial V^{\prime} / \partial q\left[\partial^{2} V / \partial q^{2}\right]^{-1} \partial V / \partial q$ is a scalar, (4.24) implies

$$
\frac{\partial V}{\partial Z} \sim \frac{\partial^{2} V}{\partial Z \partial q}\left[\frac{\partial^{2} V}{\partial q^{2}}\right]^{-1} \frac{\partial V}{\partial q}
$$

By inverting equation (4.19), we have:

$$
q \sim\left[\frac{\partial^{2} V}{\partial q^{2}}\right]^{-1} \frac{\partial V}{\partial q}
$$

so that equation (4.25) becomes

$$
\frac{\partial V}{\partial Z} \sim \frac{\partial^{2} V}{\partial Z \partial q} q
$$

But

$$
\begin{aligned}
\frac{\partial^{2} V}{\partial Z \partial q} q & =\frac{\partial}{\partial Z}\left(\frac{\partial V}{\partial H} \cdot \frac{\partial H^{\prime}}{\partial q} q\right) \\
& =\frac{\partial}{\partial Z}\left(\frac{\partial V}{\partial H} \cdot H\right) \quad \text { by homogeneity of degree one of } H \\
& =\left[\frac{\partial^{2} V}{\partial H^{2}} \cdot H+\frac{\partial V}{\partial H}\right] \frac{\partial H}{\partial Z} \\
& \sim \frac{\partial V}{\partial Z}
\end{aligned}
$$

so that equation (4.15) and the first part of equation (4.16) are satisfied. Homogeneity of degree zero of $\left(\partial H / \partial Z_{i}\right) /\left(\partial H / \partial Z_{1}\right)$ in $w$ implies that

(4.29) $\frac{\partial^{2} H}{\partial Z_{i} \partial w} w=\gamma \frac{\partial H}{\partial Z_{i}}$

$$
(i=1,2, \ldots, n)
$$

where $\gamma$ is a scalar. Thus:

$$
\begin{aligned}
\frac{\partial^{2} V}{\partial Z \partial w} w & =\frac{\partial H}{\partial Z} \frac{\partial^{2} V}{\partial H \partial w} w+\frac{\partial V}{\partial H} \frac{\partial^{2} H}{\partial Z \partial w} w \\
& =\frac{\partial H}{\partial Z} \frac{\partial^{2} V}{\partial H \partial w} w+\gamma \frac{\partial V}{\partial H} \frac{\partial H}{\partial Z}
\end{aligned}
$$

which is proportional to $\partial V / \partial Z$. Hence equation (4.16) is also satisfied. Q.E.D. 
Note that the conditions of Theorem 2 do not imply the conditions of Theorem 1. We may also add that in Theorem 2, if $H$ were "groupwise inclusively homothetic" ${ }^{\circ}$ in $w$, that is,

$$
\frac{\partial H^{\prime}}{\partial w} w=f(H)
$$

then it satisfies conditions (i) and (ii). We now present two special cases satisfying the conditions of Theorem 2, which may be of practical interest.

CASE 1: $V=V(w, q, Z)$ is homogeneous of degree $-k_{1}$ in $w$ and $-k_{2}$ in $q$. To prove that this case satisfies the conditions of Theorem 2, note that by homogeneity of degree $-k_{2}$ in $q$, one can write

$$
V(w, q, Z)=H(w, q, Z)^{-k_{2}}
$$

where $H$ is homogeneous of degree one in $q$. Moreover, homogeneity of degree $-k_{1}$ of $V$ in $w$ implies that $H(w, q, Z)$ is homogeneous of degree $k_{1} / k_{2}$ in $w$, which in turn implies that $\partial H / \partial q$ and $\partial H / \partial Z$ are both homogeneous of degree $k_{1} / k_{2}$ in $w$ and therefore the ratios, $\left(\partial H / \partial q_{i}\right) /\left(\partial H / \partial q_{1}\right)$ and $\left(\partial H / \partial Z_{i}\right) /\left(\partial H / \partial Z_{1}\right)$, are all homogeneous of degree zero.

CASE 2: $V=V(w, H(q, Z))$ where $H$ is homogeneous of degree one in $q$. It is easy to verify that the conditions of Theorem 2 are satisfied. The direct utility function corresponding to this indirect utility function is $U(L, H(X, Z))$ where $H$ is a homogeneous function in $X$.

Finally, we consider the widely used case of a utility function linear in a single nontaxable good $L$. This case, which implies zero income effects in the demands for private goods, $X$, has been widely discussed in the taxation literature (see, for example, Atkinson and Stiglitz [2]). We shall prove the following:

THEOREM 3: Suppose that the direct utility function is linear in a single nontaxable commodity L. Then condition (3.6) holds at the optimum (4.1)-(4.3) with optimum taxation if and only if $\left(\partial U / \partial Z_{i}\right) /\left(\partial U / \partial Z_{1}\right), i=2,3, \ldots, m$, is homogeneous of degree zero in $X$.

Proof: It is well known that in this case the marginal utility of income for the consumer is $1 / w$, independent of $q$ and $Z$. The indirect utility function has the form:

$$
U\left(X_{1}\left(\frac{q}{w}, Z\right), \ldots, X_{n}\left(\frac{q}{w}, Z\right), Z\right)-\sum_{i} \frac{q_{i}}{w} X_{i}\left(\frac{q}{w}, Z\right)+\frac{1}{w} .
$$

From Roy's Identity,

$$
\begin{aligned}
& \frac{\partial X}{\partial q}=-w \frac{\partial^{2} V}{\partial q^{2}} \\
& \frac{\partial X}{\partial Z}=-w \frac{\partial^{2} V}{\partial Z \partial q}
\end{aligned}
$$

\footnotetext{
${ }^{6}$ See Jorgenson and Lau $[8]$.
} 
In order for $\partial V / \partial Z$ to be proportional to $r$ at the optimum, whatever the value of $r$, equation (4.10) must hold, which implies that

$$
\begin{aligned}
\frac{\partial V}{\partial Z} & \sim \frac{\partial X}{\partial Z}\left[\frac{\partial X}{\partial q}\right]^{-1} X \\
& =-w \frac{\partial^{2} V}{\partial Z \partial q}\left[-w \frac{\partial^{2} V}{\partial q^{2}}\right]^{-1}\left(-w \frac{\partial V}{\partial q}\right) \\
& \sim \frac{\partial^{2} V}{\partial Z \partial q}\left[\frac{\partial^{2} V}{\partial q^{2}}\right]^{-1} \frac{\partial V}{\partial q}
\end{aligned}
$$

We note that

$$
\begin{aligned}
\frac{\partial^{2} V}{\partial Z \partial q} & =\frac{\partial^{2} U}{\partial Z \partial X} \frac{\partial X^{\prime}}{\partial q}+\frac{\partial^{2} U}{\partial Z \partial L} \frac{\partial L^{\prime}}{\partial q} \\
& =\frac{\partial^{2} U}{\partial Z \partial X} \frac{\partial X^{\prime}}{\partial q}, \quad \text { since } \frac{\partial^{2} U}{\partial Z \partial L}=0
\end{aligned}
$$

Thus

$$
\frac{\partial^{2} V}{\partial Z \partial q}\left[\frac{\partial^{2} V}{\partial q^{2}}\right]^{-1} \frac{\partial V}{\partial q}=\frac{\partial^{2} U}{\partial Z \partial X} X
$$

Equation (4.10) therefore implies and is implied by

$$
\frac{\partial^{2} U}{\partial Z \partial X} X=\psi \frac{\partial U}{\partial Z}
$$

where $\psi$ is a scalar. By (4.39), $\left(\partial U / \partial Z_{i}\right) /\left(\partial U / \partial Z_{1}\right)$ is homogeneous of degree zero in $X$.

Q.E.D.

\section{THE CASE OF DIRECT ADDITIVITY}

Let the direct utility function be given by

$$
U(L, X, Z)=\sum_{i=1}^{n} f_{i}\left(X_{i} \Phi_{i}(Z)\right)+L
$$

where $L$ is the quantity of a single nontaxable commodity and $\Phi_{i}$ 's are realvalued functions of $Z$. We seek conditions on the $f_{i}(\cdot)$ 's, if any, such that equation (4.14) holds for the indirect utility function corresponding to $U(L, X, Z)$. Since $U(L, X, Z)$ is linear in a single nontaxable commodity $L$, Theorem 3 applies. Thus, we seek conditions under which equation (4.39) holds. 
By direct computation,

$$
\begin{aligned}
& \frac{\partial U}{\partial Z}=\sum_{i=1}^{n} f_{i}^{\prime}\left(X_{i} \Phi_{i}(Z)\right) X_{i} \frac{\partial \Phi_{i}(Z)}{\partial Z}, \\
& \begin{aligned}
\frac{\partial^{2} U}{\partial Z \partial X} X=\sum_{i=1}^{n} f_{i}^{\prime}\left(X_{i} \Phi_{i}(Z)\right) X_{i} \frac{\partial \Phi_{i}(Z)}{\partial Z} & \\
& +\sum_{i=1}^{n} f_{i}^{\prime \prime}\left(X_{i} \Phi_{i}(Z)\right) X_{i}^{2} \Phi_{i}(Z) \frac{\partial \Phi_{i}(Z)}{\partial Z} .
\end{aligned}
\end{aligned}
$$

In order for

$$
\frac{\partial^{2} U}{\partial Z \partial X} X=\psi \frac{\partial U}{\partial Z}
$$

it is necessary to have:

$$
\sum_{i=1}^{n} f_{i}^{\prime \prime}\left(X_{i} \Phi_{i}(Z)\right) X_{i}^{2} \Phi_{i}(Z) \frac{\partial \Phi_{i}(Z)}{\partial Z}=\psi^{*} \sum_{i=1}^{n} f_{i}^{\prime}\left(X_{i} \Phi_{i}(Z)\right) X_{i} \frac{\partial \Phi_{i}(Z)}{\partial Z},
$$

where $\psi^{*}$ is a scalar function. But equation (5.5) must hold for arbitrary values of $X$, hence each term in the sum on the left-hand side must be separately proportional to the corresponding term in the sum on the right-hand side, resulting in:

$$
\frac{f_{i}^{\prime \prime}\left(X_{i} \Phi_{i}(Z)\right) X_{i} \Phi_{i}(Z)}{f_{i}^{\prime}\left(X_{i} \Phi_{i}(Z)\right)}=\psi^{*} \quad(i=1,2, \ldots, n) .
$$

Since the left-hand side of each of the equations (5.6) depends only on $X_{i}, \psi^{*}$ cannot be a function of $X$. But $X_{i} \Phi_{i}(Z)$ appears as a single variable in each of these equations, and thus $\psi^{*}$ cannot be a function of $Z$ either. So it must be a constant, say $k$. By a change of variables $y=X_{i} \Phi_{i}(Z)$, equation (5.6) may be rewritten as:

$$
\frac{f_{i}^{\prime \prime}(y) y}{f_{i}^{\prime}(y)}=k
$$

$$
(i=1,2, \ldots, n)
$$

which may be successively integrated to:

(5.8) $\quad \ln f_{i}^{\prime}(y)=k \ln y+C_{1 i}$

$(i=1,2, \ldots, n)$,

(5.9) $\quad f_{i}^{\prime}(y)=e^{C_{\text {li }} y^{k}}$

$(i=1,2, \ldots, n)$,

and

$$
f_{i}(y)=\frac{e^{C_{1 i} y^{k+1}}}{k+1}+C_{2 i}, \quad k \neq-1
$$$$
(i=1,2, \ldots, n)
$$

(5.11) $f_{i}(y)=e^{C_{1 i}} \ln y+C_{2 i}, \quad k=-1$

$(i=1,2, \ldots, n)$ 
where $C_{1 i}$ and $C_{2 i}$ are constants of integration. Hence each $f_{i}(\cdot)$ must have a power function form with a common power across all $i, i=1,2, \ldots, n$, or the logarithmic form.

We conclude that the direct utility function must have the form:

$$
U(L, X, Z)=\sum_{i=1}^{n} \frac{C_{i}^{*}\left[X_{i} \Phi_{i}(Z)\right]^{k+1}}{k+1}+L+C^{*}, \quad k \neq-1,
$$

or

$$
U(L, X, Z)=\sum_{i=1}^{n} C_{i}^{*} \ln \left[X_{i} \Phi_{i}(Z)\right]+L+C^{*},
$$

where $C_{i}^{*}=e^{C_{1 i}}$ and $C^{*}=\sum_{i=1}^{n} C_{2 i}$ are constants. One may verify directly that the utility function in equation (5.12) does not satisfy equation (3.17) for an arbitrary choice of $\Phi_{i}(Z)$ 's. Hence efficiency does not obtain in general when taxes are not optimally chosen.

\section{Stanford University and \\ Hebrew University}

Manuscript received April, 1976; final revision received February, 1977.

\section{REFERENCES}

[1] Atkinson, A. B., And N. Stern: "Pigou, Taxation and Public Goods," Review of Economic Studies, 41 (1974), 119-128.

[2] Atkinson, A. B., AND J. E. Stiglitz: "The Design of Tax Structure: Direct vs. Indirect Taxation," Journal of Public Economics, 6 (1976), 55-76.

[3] BorteuX, M.: "Sur la Gestion des Monopoles Publics Astraints à l'Equilibre Budgetaire," Econometrica, 24 (1956), 22-40.

[4] Diamond, P. A., AND J. A. Mirrlees: "Optimal Taxation and Public Production I-II," American Economic Review, 61 (1971), 8-27, 261-278.

[5] Goldman, S. M., And H. Uzawa: "A Note on Separability in Demand Analysis," Econometrica, 32 (1964), 387-398.

[6] Green, J., AND J. J. LAfFont: "On the Revelation of Preferences for Public Goods," Journal of Public Economics, 8 (1977), 79-93.

[7] Groves, T., And J. Ledyard: "Optimal Allocation of Public Goods: A Solution to the 'Free-Rider' Problem," Econometrica, 45 (1977), 783-809.

[8] Jorgenson, D. W., AND L. J. LAU: "The Structure of Consumer Preferences," Annals of Economic and Social Measurement, 6 (1975), 49-101.

[9] Musgrave, R. M.: The Theory of Public Finance. New York: McGraw-Hill, 1959.

[10] Pigou, A. C.: A Study in Public Finance, third edition. London:McMillan, 1947.

[11] RAMSEY, F. P.: "A Contribution to the Theory of Taxation," Economic Journal, 37 (1927), 47-61.

[12] RoY, R.: De l'Utilité. Paris: Hermann, 1942.

[13] Samuelson, P. A.: "The Pure Theory of Public Expenditure," Review of Economics and Statistics, 36 (1954), 387-389.

[14] Stiglitz, J. E., AND P. Dasgupta: "Differential Taxation, Public Goods and Economic Efficiency," Review of Economic Studies, 38 (1971), 151-174. 